\title{
Estrategias de marketing de marcas globales y locales en economías en desarrollo: un estudio comparativo en el mercado de chocolate de Ecuador
}

\author{
Domingo Calvo Dopico ${ }^{\mathrm{a}}$ / Ángel María del Castillo Puente ${ }^{\mathrm{b}}$ / Salomón Roberto Arias Montero ${ }^{\mathrm{c}^{*}}$ \\ a Universidade da Coruña, Departamento de Empresa, Área de Comercialización e Investigación de Mercados, Campus de \\ Elviña s/n, 15071 A Coruña, España \\ b EAE Business School, Campus de Madrid, Calle Joaquín Costa, 41, 28002 Madrid, España \\ c Universidad Técnica de Machala, Facultad de Ciencias Empresariales, Avenida 25 de junio, km 51/2 Vía Pasaje, 070150 \\ Machala, Ecuador
}

Recibido: 6 de julio de 2021 / Aceptado: 30 de septiembre de 2021

\begin{abstract}
Resumen
El objetivo de este trabajo es analizar las estrategias de marca internacional en el mercado del chocolate para un país en vías de desarrollo como es el caso de Ecuador. Se destacan dos resultados de interés. En primer lugar, a pesar de tratarse de un país de gran tradición cacaotera, lo cual predispone a una preferencia por productos o marcas locales, Nestlé lidera el mercado del chocolate. Los principales elementos que explican este liderazgo se fundamentan en la implantación de una estrategia híbrida que ha sabido combinar un posicionamiento global para todos los mercados en los que opera con la adaptación de sus programas de marketing al mercado local (Ecuador). En segundo lugar, Nestlé, además de conseguir el reconocimiento de una marca global, conocida y, por tanto, de gran notoriedad, ha sabido adaptarse a una economía en desarrollo a través de la fijación de precios competitivos, una importante actividad promocional, una estrategia de distribución intensiva y una amplia gama que también ofrece nuevos productos para diferentes segmentos como el chocolate blanco con arroz crocante o el chocolate elaborado a partir de cacao fino de Ecuador.
\end{abstract}

\section{Palabras clave}

Marca global; marca local; chocolate; estrategias de marketing; economías en desarrollo.

\section{Marketing strategies of global and local brands in developing economies: a comparative study in the Ecuadorian chocolate market}

\begin{abstract}
The aim of this paper is to analyze international brand strategies in the chocolate market for a developing country as Ecuador. Two interesting results stand out. Firstly, despite being a country with a great cocoa tradition, which predisposes to a preference for local products or brands, Nestlé leads the chocolate market. The main elements that explain this leadership are based on the implementation of a hybrid strategy that has managed to combine a global positioning for all the markets in which it operates with the adaptation of its marketing programmes to the local market (Ecuador). Secondly, Nestlé, in addition to having achieved the recognition of a global, well known and, therefore, a great notoriety brand, was able to adapt to a developing economy through competitive pricing, significant promotional activity, an intensive distribution strategy and a wide range of products that also offers new products for different segments such as white chocolate with crispy rice or chocolate made from fine cocoa from Ecuador.
\end{abstract}

\section{Keywords}

Global brand; local brand; chocolate; marketing strategies; developing economies.

JEL Codes: M16, M31.

* D. Calvo: domingo.calvo.dopico@udc.es; A. M. del Castillo: acastillo@eae.es; S. R. Arias (autor para correspondencia): sarias@utmachala.edu.ec 


\section{Introducción}

La creciente globalización de los mercados agroalimentarios representa un fenómeno que cada vez es más interesante investigar. Aunque existe una oportunidad para las marcas denominadas domésticas o locales de expandirse a otros mercados, también entran en escena marcas con un alto grado de internacionalización, denominadas marcas globales, que han fundamentado su estrategia de crecimiento en vender sus productos actuales en nuevos mercados. Es el caso de la multinacional Nestlé, que se ha introducido en un país de gran tradición cacaotera como es Ecuador. Este país es uno de los grandes productores de cacao del mundo, que además produce una materia prima de excelente calidad situada en una superficie de más de 600.000 hectáreas (Instituto Nacional de Estadística y Censos [INEC], 2020). De acuerdo con la Asociación Nacional de Exportadores de Cacao e Industrializados del Ecuador [ANECACAO], 2019), de la totalidad que se destina a la exportación, aproximadamente el 91\% se centra en el grano de cacao, que es la materia prima demandada por la industria a escala mundial; el $8 \%$ se destina a productos semielaborados como pasta, manteca o polvo, mientras que solo el $1 \%$ de las exportaciones está dedicado a la exportación del chocolate.

La compra y el consumo de productos de alimentación están influidos por la tradición, hábitos, costumbres y cultura (Fernández-Ferrín, Calvo-Turrientes, Bande, Artaraz-Miñón y Galán-Ladero, 2018). Además, Le, Thi Nguyen y van Nguyen (2013) han demostrado que la identidad nacional tiene un impacto positivo sobre la evaluación del producto y el etnocentrismo del consumidor. Así, para un determinado territorio o área geográfica existe un vínculo, moderado por el etnocentrismo, entre los consumidores y los productos locales, que predispone favorablemente a la compra o a la demanda de estos productos. Por ello, cuando la oferta comercial de una marca se ajusta a los valores, a la tradición o a la cultura de un determinado territorio, se acrecienta la preferencia por estos productos. De igual manera, Schuiling y Kapferer (2004) explican las ventajas estratégicas de las marcas locales, las cuales tienen mejor acceso a las materias primas e incluso pueden responder a las necesidades no cubiertas por las marcas internacionales. Por otro lado, el consumo local tiene beneficios ambientales, sociales y económicos. Por tanto, estas ventajas podrían favorecer que se produzca una mayor demanda de las marcas locales.

Sin embargo, existen muchas ventajas para las marcas globales, entre las que se encuentran las economías de escala. Una marca estandarizada genera importantes reducciones de costes de comercialización, compras de materia prima, fabricación y logística. De igual manera, Winit, Gregory, Cleveland y Verlegh (2014) sostienen que las marcas globales pueden operar en diferentes mercados geográficos con un mismo o similar posicionamiento. Gracias a esta posición global y a las economías de escala en la fabricación o comercialización y estandarización, se generan ahorros sustanciales en los costes (Craig y Douglas, 2000; Levitt, 1983) que permiten ofrecer precios más reducidos. Otra ventaja es el desarrollo de una imagen de marca única en todos los países, aspecto que es especialmente importante en ciertas categorías de productos cuyas marcas se dirigen a segmentos de consumidores globales (Hassan y Katsanis, 1991).

Además, la globalización de los mercados está influyendo en el comportamiento de compra de los consumidores, puesto que el mayor grado de notoriedad de las marcas globales hace que estas pasen a formar parte del denominado conjunto evocado, es decir, del conjunto de alternativas que entran a formar parte de la mente del consumidor, perfilándose como una alternativa con mayor probabilidad de compra. Por otro lado, la investigación previa que ha abordado la influencia de las marcas (Aaker, 1991; Keller, 1993) ha demostrado que la notoriedad y la imagen de marca son factores determinantes en la creación de una posición predominante en la mente del consumidor. De esta manera, existe una relación significativa entre la consciencia de marca y la elección o compra. A partir de este hallazgo se deduce que las asociaciones positivas que las marcas globales generan en la mente del consumidor refuerzan todavía más la preferencia por estas marcas frente a sus competidoras. Las ventajas de construir marcas internacionales y globales, amparadas en una estrategia de marketing global, han sido frecuentemente enfatizadas por Levitt (1983) y Kapferer (2005), entre otros. Ahora bien, 
Aaker y Joachimsthaler (1999) destacan que es necesario que las empresas realicen un proceso de planificación de marca global común, que sea consistente en todos los mercados y que sea capaz de crear estrategias coherentes de construcción de capital de marca. Chabowski, Samiee y Hult (2013) han aportado un marco teórico que integra las decisiones estratégicas que debe de acometer la empresa (estandarización, adaptación o extensión) como su influencia en el desarrollo de la marca, también denominado global branding. Finalmente, es posible medir el resultado o desempeño de esas actividades comerciales que se han puesto en marcha en un determinado país o región a través del análisis de la performance, utilizando indicadores como la probabilidad de recompra, la cuota de mercado o el brand equity.

Ante esta disyuntiva de marcas locales y globales, es importante investigar si en una economía en desarrollo estas marcas pueden no solo competir en estos mercados sino llegar a ocupar una posición de liderazgo consolidado. Dada la escasez de estudios empíricos que han abordado esta cuestión, consideramos de gran interés proporcionar evidencia empírica sobre esta cuestión de suma importancia económica y empresarial. Así, uno de los objetivos de este trabajo es analizar el grado de estandarización o adaptación de la estrategia de la marca internacional y el desarrollo de la marca, o global branding, del mercado de chocolate de Ecuador. Para el análisis de esta cuestión, se incorpora no solo el análisis de la empresa, sino que se complementa con el punto de vista del consumidor, aspecto cada vez más importante en los estudios de globalización (Steenkamp, Batra y Alden, 2003). El otro objetivo es saber el impacto que esas estrategias de marca tiene en el resultado empresarial o performance, la cual puede ser medida mediante la cuota de mercado, la notoriedad y el valor de marca, también denominado brand equity.

La contribución de este estudio es notable. La investigación de este escenario permite avanzar en los escasos estudios empíricos sobre marketing internacional y valor de marca que analizan el fenómeno de las marcas globales en economías en vías de desarrollo, donde, además, se integra tanto el punto de vista del consumidor, o de la demanda, con el de la oferta, o empresa.

Este trabajo de investigación se ha estructurado como sigue. Primeramente, se formula el marco teórico y se comenta la investigación previa directamente relacionada con las marcas globales y locales. A continuación, se explica la metodología. Posteriormente, a partir de los conceptos de marcas globales, locales, branding e investigación previa -que para el caso de países en vías de desarrollo es escaso-, se analizan los resultados. Finalmente, se discuten los resultados y se formulan las conclusiones, destacando también las limitaciones y las futuras líneas de investigación.

\section{Marco teórico. Conceptualización y revisión de la literatura}

En primer lugar, se explica lo qué se entiende por marcas globales y locales para, posteriormente, centrarnos en el modelo teórico que analiza las estrategias de marca internacional.

\subsection{Marcas globales y marcas locales}

La literatura ha concretado la definición de la marca global. Siguiendo a Dimofte, Johansson y Ronkainen (2008), se puede concluir que las marcas globales son aquellas que están disponibles en numerosos mercados internacionales $\mathrm{y}$, además, ostentan un alto nivel de reconocimiento. Por su parte, Aaker y Joachimsthaler (1999) explican que las marcas globales presentan un posicionamiento, personalidad y publicidad similar en todos los mercados donde una empresa opera. También existe un punto de vista que ha analizado la globalidad de la marca desde la perspectiva de las percepciones del consumidor. Así, Özsomer y Altaras (2008) definen las marcas globales como aquellas que tienen un conocimiento, disponibilidad, aceptación y demanda regional o global generalizada y, a menudo, se encuentran bajo el mismo nombre. Sin embargo, las marcas locales están disponibles geográficamente en un país o en una área geográfica determinada, aunque pueden ser propiedad de una empresa lo- 
cal, internacional o global (Schuiling y Kapferer, 2004). Por tanto, la marca global se define principalmente sobre la base del aspecto geográfico, mientras que la definición de marca local pone el énfasis en la distribución geográfica y en la propiedad. Por su parte, la empresa local puede encontrar su ventaja competitiva en la explotación del posicionamiento basado en el lugar de origen del producto.

\subsection{Estrategias de marca internacional, global branding y desempeño}

De acuerdo con Chabowski, Samiee y Hult (2013), la estrategia influye en la configuración de capacidades que, a su vez, impacta en el desempeño de la empresa. Así, la estrategia de marca internacional (adaptación, estandarización o extensión de marca) influye en el posicionamiento de la marca (producto, promoción, distribución o precio), marca/país de origen (local o no local), e imagen-concepto de marca (funcional, experiencial o simbólica). Este conjunto es lo que se denomina desarrollo de capacidades basadas en la marca. Del mismo modo, la influencia combinada de estas variables afecta el desempeño de la marca (participación de mercado, probabilidad de compra o valor de marca). La Figura 1 sintetiza estas relaciones. A continuación, se describe de manera sintética cada uno de los apartados.

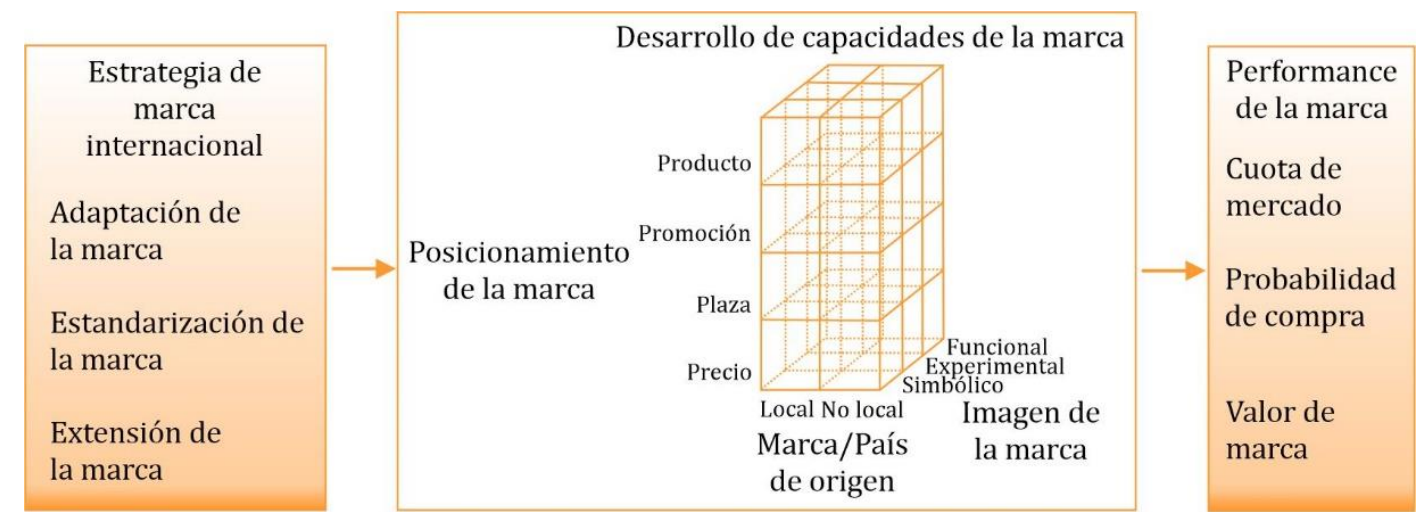

Figura 1. Marco teórico para investigar la estrategia de marca internacional. Nota: el desarrollo de las capacidades de la marca (cubo central) incorpora tres dimensiones: (1) posicionamiento de la marca (cuatro niveles), (2) concepto-imagen de marca (tres niveles), y (3) marca/país de origen (dos niveles). Fuente: Chabowski, Samiee y Hult (2013).

\subsubsection{Estrategia de marca internacional. Estandarización vs. adaptación y su influencia en el desarrollo de la marca}

Una parte importante de la investigación de la marca global se dedica a la discusión sobre adaptación y estandarización (Aaker y Joachimsthaler, 1999; Levitt, 1983). Mientras que Levitt (1983) defiende una completa estandarización de la estrategia de la marca y de los elementos del marketing-mix cuando se define una marca global, Kapferer (2005) señala que las marcas no se pueden entender como un modelo de estandarización total, sino que deben adaptar sus productos a los mercados domésticos en función de las percepciones de los mercados en los que opera la marca. Esta adaptación oscila entre una ligera modificación del producto, o en la estrategia de marketing, hasta ofrecer una versión del producto diferente (Cerviño Fernández y Cebollada Calvo, 2005). Chabowski, Samiee y Hult (2013) destacan una estrategia híbrida, la cual da como resultado una configuración del producto, de la distribución y de la fijación de precios que debe adaptarse a las preferencias del mercado local. Por ello, el objetivo es adaptar la oferta de la empresa a las preferencias de los clientes y a las condiciones del mercado situado en el exterior. 


\subsubsection{Desarrollo de la marca}

El posicionamiento de la marca es otro componente clave en la investigación de la marca global. Estos autores destacan principalmente la publicidad y la promoción internacional, las cuales, apoyándose en iconos culturales, se configuran como buenas herramientas de posicionamiento de la marca a nivel global. Un ejemplo excelente de cómo el uso de iconos culturales contribuye a la creación de este posicionamiento global lo ha constituido la marca Nespresso, que ha conseguido posicionarse en el mercado de cápsulas de café como una marca de gran reconocimiento a nivel mundial. Junto a esta variable también destacan las variables del producto, distribución y precio.

Otro de los elementos fundamentales identificado en la investigación de la marca global es la marca país de origen. En relación con la configuración de la cartera de marcas de las empresas en el mercado, destacan principalmente el etnocentrismo del consumidor (Shimp y Sharma, 1987), la actitud hacia las marcas globales y la marca del país de origen (Kilduff y Núñez Tabales, 2017). De aquí ha surgido un concepto de gran interés para explicar la influencia que ejercen las marcas globales: la globalidad percibida de la marca (perceived globalness brand). Así, Steenkamp, Batra y Alden (2003) explican que, en la medida en que se produzca una percepción de una mayor presencia en los mercados y en la medida en que el reconocimiento de una marca se incremente, la percepción de la globalidad también aumentará. De igual manera, también surge la actitud opuesta, la animosidad del consumidor, la cual influye negativamente en la orientación al consumo global (Klein, 2002).

Finalmente, nos encontramos con el concepto-imagen de marca. Directamente vinculado con este concepto se encuentran la dimensión funcional, experiencial y simbólica, o significado de las marcas. Específicamente, el uso funcional de una marca resuelve las compras básicas o rutinarias del consumidor, mientras que el uso experiencial es de naturaleza sensorial. En lo que se refiere a la dimensión simbólica, esta se centra en el imaginario del consumidor.

\subsubsection{Desempeño empresarial e influencia del desarrollo de marca}

El último componente pone el énfasis en el desempeño de la marca, que tiene diferentes indicadores entre los que destacan el valor de marca, el reconocimiento de la marca, la cuota de mercado y la probabilidad de compra (reintención de compra). La teoría del valor de marca (Aaker, 1996a; Keller, 2003) sostiene que si la decisión del consumidor está condicionada por el precio, el desarrollo de la capacidad de la marca debería estar más centrado en la consecución de una mayor cuota de mercado, o una mayor probabilidad de compra, que en conseguir un mayor valor de marca. Alternativamente, si la decisión del consumidor no viene condicionada por el precio, el desarrollo de la capacidad de marca se podría focalizar más en conseguir un mayor valor de marca a través de valores distintivos, como la superioridad funcional, una gran personalidad de marca, la personalización, o experiencias únicas, etcétera.

\subsubsection{Evidencia empírica. Marcas globales vs. marcas locales}

En un proyecto de investigación con 3.300 consumidores en 41 países, Taylor, Quelch y Holt (2004) descubrieron que la mayoría de los consumidores prefieren una marca global sobre otra debido a las diferencias en las cualidades de las marcas globales frente a las locales. Sin embargo, para el caso de los productos agroalimentarios, la preferencia viene condicionada por el precio y el etnocentrismo de los consumidores (Winit, Gregory, Cleveland y Verlegh, 2014) y por el grado de heterogeneidad o fragmentación del mercado. Así, Rajh, Vranesevic y Tolic (2003) analizaron el valor de marca en la industria alimentaria de la República de Croacia para cinco categorías de productos como las bebidas carbonatadas (refrescos), el chocolate, el café, la cerveza y la leche. El estudio concluyó que las marcas locales son más preferidas que las marcas globales en el caso de la leche y la cerveza -categorías donde puede existir una actitud más etnocentrista-, mientras que en el mercado del chocolate y de las bebidas refrescantes las marcas globales son las preferidas. De igual manera, Öszomer (2012) demuestra que, en economías en desarrollo, donde existan mercados altamente fragmentados 
y heterogéneos, la preferencia en productos de alimentación básicos se inclina más por productos locales.

En cuanto a las variables que más influyen sobre el brand equity o el valor de marca destacan la calidad percibida y la imagen de marca, que ejercieron una influencia positiva en la intención de compra del pan, estudio que fue realizado en Java Oriental (Indonesia) por Harsono, Perdana, Riyadi y Normasita (2018). De nuevo, en otra economía en desarrollo como Senegal, Demont et al. (2013) concluyeron que los consumidores urbanos estarían dispuestos a pagar una prima de precio por arroz de calidad certificado por la marca (p. ej., marca Rival ${ }^{\circledR}$ ).

En lo que se refiere al mercado del chocolate, se ha constatado la escasez de estudios donde se pueda comprobar la comparativa del valor de marca entre marca global y local, y donde solo se ha encontrado el estudio ya comentado de Rajh, Vranesevic y Tolic (2003). En la revisión de estudios realizados sobre esta categoría (Lybeck, Holmlund-Rytkönen y Sääksjärvi, 2006; Ozretic-Dosen, Skare y Krupka, 2007; Poelmans y Rousseau, 2016; Rousseau, 2015; Thaichon, Jebarajakirthy, Tatuu y Gautam Gajbhiyeb, 2018) se ha confirmado que el chocolate es consumido principalmente por motivos hedónicos, donde el sabor desempeña un papel determinante. Otros resultados que hay que destacar han sido la importancia que tiene el reconocimiento de la marca, el precio o la acción promocional en la recompra del producto y cómo el consumidor se mantiene fiel a la marca.

\section{Estudio empírico}

A partir del marco teórico y de la revisión de la literatura, se va a proceder a realizar un estudio empírico para el caso del mercado de chocolate de Ecuador, donde opera la marca global Nestlé y el resto de las marcas locales (Universal, Pacari, República de Ecuador y Confiteca). Este estudio empírico contiene los objetivos, la metodología y los resultados de la investigación de mercado.

\subsection{Objetivos}

Los objetivos son dos. En primer lugar, se quiere investigar el grado de estandarización o adaptación de la estrategia de marca y del desarrollo de la marca (global branding) en el mercado de Ecuador. Para el análisis de esta cuestión, se incorpora no solo el análisis de la empresa, sino que se complementa con el punto de vista del consumidor, aspecto cada vez más importante en los estudios de globalización (Steenkamp, Batra y Alden, 2003). El otro objetivo es saber el impacto que el desarrollo de marca, o global branding, tiene en el resultado empresarial o performance. Para la medición de la performance se recurrirá a analizar la cuota de mercado, la notoriedad de la marca y el valor de marca, también denominado brand equity.

\subsection{Metodología. Fuentes de información, variables y medición}

La metodología combina tanto la investigación cualitativa como la investigación cuantitativa. La investigación cuantitativa consiste en una investigación de mercados que analiza los hábitos de compra por categoría de producto, la importancia de la marca (notoriedad y fuentes de valor) y la cuota de mercado. Se ha realizado un cuestionario personal. Un detalle de la fuente de información, las variables y escalas de medida en relación con cada objetivo puede verse en la Tabla 1.

Tabla 1. Fuente de información, variables analizadas en este estudio y escalas utilizadas

\begin{tabular}{llll}
\hline $\begin{array}{l}\text { Fuente de } \\
\text { información }\end{array}$ & Variable analizada & Escala utilizada & Referencias bibliográficas \\
\hline $\begin{array}{l}\text { Cuestionario } \\
\text { personal }\end{array}$ & $\begin{array}{l}\text { Hábitos de compra por categoría de compra } \\
\text { (chocolate negro, chocolate blanco, almen- } \\
\text { dra, bombón o productos de confitería) }\end{array}$ & $\begin{array}{l}\text { Frecuencias absolutas de compra } \\
\text { (escala clásica que combina las } \\
\text { variables que se quieren medir) }\end{array}$ & $\begin{array}{l}\text { Calvo Dopico, del Castillo } \\
\text { Puente Herrera Peña } \\
(2020)\end{array}$ \\
\hline
\end{tabular}


Tabla 1 (continuación). Fuente de información, variables analizadas en este estudio y escalas utilizadas

\begin{tabular}{llll}
\hline $\begin{array}{l}\text { Fuente de } \\
\text { información }\end{array}$ & Variable analizada & Escala utilizada & Referencias bibliográficas \\
\hline $\begin{array}{l}\text { Cuestionario } \\
\text { personal }\end{array}$ & $\begin{array}{l}\text { Notoriedad (reconocimiento de la marca) } \\
\text { de marca habitualmente comprada }\end{array}$ & Frecuencia absoluta & Aaker (1996a) \\
& $\begin{array}{l}\text { Fuentes del valor de marca (notoriedad, ca- } \\
\text { lidad percibida, lealtad y asociaciones de } \\
\text { marca) y disposición a pagar }\end{array}$ & Escala tipo Likert (5 puntos) & $\begin{array}{l}\text { Yoo, Donthu y Lee (2000); } \\
\text { Coo y Donthu (2001) }\end{array}$ \\
& Cuota de mercado & $\begin{array}{l}\text { Variable proxy: tasa de penetra- } \\
\text { ción (marca de productos de } \\
\text { chocolate que habitualmente } \\
\text { compran los consumidores) }\end{array}$ & $\begin{array}{l}\text { Romaniuk, Dawes y } \\
\text { Nenycz-Thiel (2018) }\end{array}$ \\
\hline
\end{tabular}

Fuente: elaboración propia.

\section{- Organización del cuestionario}

El cuestionario se ha organizado de acuerdo con la siguiente estructura. El primer apartado se centra en analizar los hábitos de compra del chocolate. Se solicitó al encuestado que indicase los hábitos de compra para las diferentes opciones de chocolate (tableta de chocolate negro, tableta de chocolate blanco, tableta de almendra, bombón o productos de confitería). En el segundo apartado del cuestionario se analizan los motivos de compra y, a continuación, los criterios de la fase de evaluación del proceso de compra. No fue necesario realizar el análisis de estas variables. El siguiente apartado se centró en el análisis de la marca, diferenciando entre la notoriedad espontánea (reconocimiento) y las fuentes del valor de marca. Para la medición de los hábitos de compra se ha utilizado una escala de frecuencias de compra (Calvo Dopico, del Castillo Puente y Herrera Peña, 2020). Para la medición de los motivos se formuló una pregunta abierta, de tal forma que se pudiera conseguir directamente del encuestado una respuesta precisa, lo que permitió obtener una buena calidad de información y evitar sesgos en el estudio. A su vez, esos atributos se contrastaron con la revisión de la literatura de los trabajos de investigación relacionados con el consumo de los productos agroalimentarios (Grunert, Baadsgaard, Larsen y Madsen, 1996; Steenkamp, 1986). Para la medición de la notoriedad espontánea de la compra de la marca habitualmente comprada se utilizó una pregunta abierta. De esta forma, el encuestado respondía espontáneamente sobre las marcas que se le venían a su mente en primer, segundo o tercer lugar. El examen de las fuentes del valor de marca (notoriedad, calidad percibida, imagen o asociaciones y lealtad), además del valor de marca y de la disposición a pagar, se fundamentó en Aaker (1996b). Para la medición del valor de marca y de la disposición a pagar, se utilizaron escalas de medida ya contrastadas en los estudios de Yoo, Donthu y Lee (2000) y Yoo y Donthu (2001), para las cuales se recurrió a una escala tipo Likert. Finalmente, se midieron las variables sociodemográficas (edad, género, renta, hábitat y tipología del hogar).

\section{- Muestreo y trabajo de campo}

La unidad muestral de este estudio se corresponde con los consumidores de chocolate de los diferentes hogares. El cuestionario se realizó de forma personal. Ante cualquier pregunta del encuestado, se aclaraban sus posibles dudas. El proceso de recogida de datos se llevó a cabo entre julio de 2016 y febrero de 2017. El tamaño muestral fue de 1.549 y el error muestral del 2,54\% (nivel de confianza del 95,5\%). Se realizó la codificación de las preguntas abiertas según la tabla de códigos que fue creada en la fase del pretest. Las características de la muestra se reflejan de forma sintética en la Tabla 2 .

La investigación cualitativa ha consistido en la visita a varios establecimientos para estudiar las variables de marketing. Esos lugares de compra son los diferentes canales que están accesibles para los 
consumidores de Ecuador, como son la tienda tradicional, Supermaxi, Mi Comisario, Aki y TIA. Se examinaron las variables relacionadas con la gama del producto, los precios, la promoción (descuentos o promociones en cantidad), la distribución comercial y la ocupación de espacio en el lineal. Para analizar el producto, se ha tenido en cuenta la amplitud de la cartera de producto. En el caso de la variable precio, se ha analizado qué estrategia de precios siguen las empresas, diferenciando entre las alternativas de estrategia basada en los costes, la competencia o la demanda. Se realizó la primera visita en mayo de 2021 y de nuevo se realizó otra en agosto de 2021 que sirvió para contrastar mejor la estrategia de precios. Gracias a este análisis ha sido posible identificar como las diferentes marcas siguen similares patrones en la fijación y establecimiento de los precios. El cálculo del precio unitario se ha redondeado a tres decimales. Para el caso de la distribución, se ha verificado si esta era intensiva o selectiva. Finalmente, se ha estudiado la variable comunicación.

Tabla 2. Estructura sociodemográfica de la muestra

\begin{tabular}{|c|c|}
\hline Edad & Porcentaje \\
\hline $\begin{array}{l}\text { Entre } 20 \text { y } 30 \text { años } \\
\text { Entre } 31 \text { y } 40 \text { años } \\
\text { Entre } 41 \text { y } 50 \text { años } \\
\text { Entre } 51 \text { y } 60 \text { años } \\
\text { Más de } 60 \text { años } \\
\text { Total }\end{array}$ & $\begin{array}{c}39,70 \% \\
31,44 \% \\
19,75 \% \\
7,75 \% \\
1,36 \% \\
100 \%\end{array}$ \\
\hline Tipo de hogar & Porcentaje \\
\hline $\begin{array}{l}\text { Con niños } \\
\text { Con adolescentes } \\
\text { Con hijos mayores } \\
\text { Solo jóvenes } \\
\text { Solo adultos } \\
\text { Jubilados } \\
\text { Total }\end{array}$ & $\begin{array}{c}30,02 \% \\
24,66 \% \\
6,26 \% \\
10,91 \% \\
27,18 \% \\
0,97 \% \\
100 \%\end{array}$ \\
\hline Renta & Porcentaje \\
\hline $\begin{array}{l}\text { Menor de } 400 \$ \\
\text { Entre } 401-800 \$ \\
\text { Entre } 800-1.200 \$ \\
\text { Entre } 1.200-1.600 \$ \\
\text { Entre } 1.600-2.000 \$ \\
\text { Más de } 2.000 \$ \\
\text { Total }\end{array}$ & $\begin{array}{c}31,96 \% \\
24,34 \% \\
18,01 \% \\
15,43 \% \\
9,68 \% \\
0,58 \% \\
100 \%\end{array}$ \\
\hline Género & Porcentaje \\
\hline $\begin{array}{l}\text { Hombre } \\
\text { Mujer } \\
\text { Total }\end{array}$ & $\begin{array}{c}49,06 \% \\
50,94 \% \\
100 \%\end{array}$ \\
\hline Hábitat & Porcentaje \\
\hline $\begin{array}{l}\text { Costa } \\
\text { Sierra } \\
\text { Oriente } \\
\text { Total }\end{array}$ & $\begin{array}{c}45 \% \\
47 \% \\
8 \% \\
100 \% \\
\end{array}$ \\
\hline
\end{tabular}

Fuente: elaboración propia.

\subsection{Análisis de resultados}

Tal y como destaca el modelo conceptual, las variables de marketing como el producto, el precio, la distribución y la comunicación son uno de los ejes determinantes para comprender el desarrollo de la 
marca. Por otro lado, su examen nos proporciona mucha información para identificar si la marca Nestlé estandariza o adapta sus programas de marketing.

\subsubsection{Análisis de las variables de marketing-mix}

En relación con la cartera de producto, se constató que la cartera de producto de Nestlé no solo se presenta como la marca con gama de mayor amplitud, sino que adapta el producto a las preferencias locales y, además, está presente en todos los canales o puntos de venta. Así, adicionalmente a las tabletas de chocolate negro y crujiente, ofrecido a través de las marcas Nestlé Clásico y Crunch, respectivamente, ofrecen el chocolate blanco a través de la marca Nestlé Galak, que contiene arroz crocante. De este modo, la marca Nestlé desarrolla una estrategia de adaptación local que ofrece productos vinculados a los hábitos de los consumidores de Ecuador. Es la única marca que ofrece chocolate blanco. Igualmente, hay otras marcas como Pacari, República del Cacao o Hershey's, con una oferta únicamente centrada en el chocolate negro. Mediante el análisis de esta variable se puede concluir que el grado de adaptación de la marca global Nestlé es muy notable, al ofrecer productos adaptados a las preferencias locales de los consumidores de Ecuador.

En referencia a los precios, en las Tablas 3, 4, 5 y 6 se observan los datos para las diferentes marcas y los distintos canales de venta. La marca local denominada La Universal presenta el chocolate negro con diferentes variedades y precios muy competitivos. Esta marca tiende a ofrecer el precio más bajo del conjunto de alternativas en los diferentes establecimientos (Mi Comisariato, Comisariato Aki, Comisariato TIA). En el caso de Supermaxi, como se puede ver, es Nestlé quien lo consigue. También ocurría esto en el análisis de los precios realizados en el mes de mayo. A pesar de esto, la marca Nestlé, gracias a una amplia oferta y variedad, parece adaptarse mejor a las preferencias del conjunto de mercado de chocolate de Ecuador. Un claro ejemplo es la gran variedad presente en Supermaxi. Otra evidencia es el chocolate blanco denominado Nestlé Galak, un chocolate dulce con arroz crocante, que cuenta también con mucha aceptación. Por ello, se deduce que Nestlé, además de desarrollar una estrategia de precios competitiva, está desarrollando nuevos productos (avellana, negro y blanco), presenta una amplia cartera de producto y tiene una presencia masiva en todos los canales de venta.

Para la tableta de chocolate negro, La Universal presenta oferta con otras variedades que incluyen el cacahuete. La marca Pacari y la marca República del Cacao, que poseen una menor cantidad de gramos, se concentran en esta categoría de la tableta de chocolate negro. Estas marcas presentan no solo un precio (\$/gr) relativamente mayor, sino también un precio más alto por unidad de venta, por lo que parece que existe, a diferencia de Nestlé y de La Universal, una apuesta más clara por posicionar el producto en un nivel superior.

En referencia a la intensidad promocional, en el caso de Nestlé también es alta. Las promociones en precio o en cantidad permiten obtener un valor percibido superior como consecuencia de un mayor valor transaccional. Por ello, se observa que la fijación de precios de la marca global Nestlé se basa en los precios de la competencia. Es decir, se trata de una estrategia de precios competitiva. También se ha detectado el uso de la promoción a través del descuento en el precio, principalmente en el Comisariato Aki.

Tabla 3. Resumen de precios de las principales marcas de chocolate por lugar de compra (Comisariato TIA)

\begin{tabular}{|c|c|c|c|c|c|}
\hline \multicolumn{3}{|c|}{ Nestlé } & \multicolumn{3}{|c|}{ La Universal } \\
\hline Variedad & $\begin{array}{l}\text { Peso } \\
\text { (gr) }\end{array}$ & $\begin{array}{l}\text { Precio de venta }(\$) \text { y } \\
\text { Precio unitario }(\$ / g r)\end{array}$ & Variedad & $\begin{array}{l}\text { Peso } \\
\text { (gr) }\end{array}$ & $\begin{array}{l}\text { Precio de venta }(\$) \text { y } \\
\text { Precio unitario }(\$ / \text { gr) }\end{array}$ \\
\hline Blanco y arroz 4U & 80 & $1,99 \$ /(0,025 \$ /$ gr $)$ & Negro y maní $4 \mathrm{U}$ & 112 & $1,99 \$ /(0,018 \$ / g r)$ \\
\hline Negro y arroz $4 U$ & 80 & $1,99 \$ /(0,025 \$ / g r)$ & Negro y blanco & 112 & $1,99 \$ /(0,018 \$ / g r)$ \\
\hline Blanco y arroz & 90 & $1,89 \$ /(0,021 \$ / g r)$ & Negro y maní* & 100 & $1,79 \$ * /(0,018 \$ / g r)$ \\
\hline Crunch & 80 & $1,99 \$ /(0,025 \$ / g r)$ & Almendras & 100 & $1,99 \$ /(0,020 \$ / \mathrm{gr})$ \\
\hline
\end{tabular}

Nota: *Precio de venta más bajo de las diferentes categorías de productos de chocolate. Fuente: elaboración propia a partir de la visita a establecimientos (2021). 
Tabla 4. Resumen de precios de las principales marcas de chocolate por lugar de compra (Supermaxi)

\begin{tabular}{|c|c|c|c|c|c|}
\hline \multicolumn{3}{|c|}{ Nestlé } & \multicolumn{3}{|c|}{ La Universal } \\
\hline Variedad & $\begin{array}{c}\text { Peso } \\
\text { (gr) }\end{array}$ & $\begin{array}{l}\text { Precio de venta }(\$) \text { y } \\
\text { Precio unitario }(\$ / g r)\end{array}$ & Variedad & $\begin{array}{l}\text { Peso } \\
\text { (gr) }\end{array}$ & $\begin{array}{l}\text { Precio de venta }(\$) \text { y } \\
\text { Precio unitario }(\$ / g r)\end{array}$ \\
\hline Negro y arroz Crunch & 90 & $1,57 \$ /(0,017 \$ / g r)$ & Negro y cacahuete L & 100 & $1,69 \$ /(0,017 \$ / g r)$ \\
\hline Negro & 100 & $1,66 \$ /(0,017 \$ / g r)$ & Negro y blanco & 112 & $1,56 \$ /(0,014 \$ / g r)$ \\
\hline Blanco y arroz & 90 & $1,571 \$ /(0,017 \$ / g r)$ & Negro y cacahuete $4 \mathrm{U}$ & 112 & $1,56 \$ /(0,014 \$ / g r)$ \\
\hline Blanco y arroz $4 \mathrm{U}$ & 80 & $1,73 \$ /(0,022 \$ / \mathrm{gr})$ & \multirow{4}{*}{\multicolumn{3}{|c|}{ No presenta más variedades en la cartera de producto. }} \\
\hline Negro y avellana & 60 & $1,89 \$ /(0,032 \$ / \mathrm{gr})$ & & & \\
\hline Negro y almendra* & 60 & $1,36 \$ * /(0,023 \$ / g r)$ & & & \\
\hline Negro y leche* & 60 & $1,36 \$ * /(0,023 \$ / \mathrm{gr})$ & & & \\
\hline \multicolumn{3}{|c|}{ Pacari } & \multicolumn{3}{|c|}{ Hershey's } \\
\hline Variedad & $\begin{array}{l}\text { Peso } \\
\text { (gr) }\end{array}$ & $\begin{array}{l}\text { Precio de venta }(\$) \text { y } \\
\text { Precio unitario }(\$ / g r)\end{array}$ & Variedad & $\begin{array}{l}\text { Peso } \\
\text { (gr) }\end{array}$ & $\begin{array}{l}\text { Precio de venta }(\$) \text { y } \\
\text { Precio unitario }(\$ / g r)\end{array}$ \\
\hline Negro (Esmeralda) & 50 & $2,01 \$ /(0,040 \$ / g r)$ & Negro & 102 & $\begin{array}{c}3,80 \$ \text { (Oferta: } 2,65 \$) / \\
(0,026 \$ / g r)\end{array}$ \\
\hline Negro (Maracuya) & 50 & $2,01 \$ /(0,040 \$ / g r)$ & Blanco & 113 & $\begin{array}{c}3,80 \text { (Oferta: } 3,23 \$) / \\
(0,029 \$ / \mathrm{gr})\end{array}$ \\
\hline Negro (Arándano) & 50 & $2,01 \$ /(0,040 \$ / g r)$ & \multicolumn{3}{|c|}{ No presenta más variedades en la cartera de producto. } \\
\hline
\end{tabular}

\begin{tabular}{lcc}
\hline & República de Ecuador \\
\hline Variedad & $\begin{array}{c}\text { Peso } \\
\text { (gr) }\end{array}$ & $\begin{array}{c}\text { Precio de venta }(\$) \text { y } \\
\text { Precio unitario }(\$ / \text { gr) }\end{array}$ \\
\hline Negro & 50 & $4,27 \$($ oferta: $2,57 \$) /$ \\
& & $(0,051 \$ /$ gr $)$
\end{tabular}

No presenta más variedades en la cartera de producto.

Nota: *Precio de venta más bajo de las diferentes categorías de productos de chocolate. Fuente: elaboración propia a partir de la visita a establecimientos (2021).

Tabla 5. Resumen de precios de las principales marcas de chocolate por lugar de compra (Mi Comisariato)

\begin{tabular}{|c|c|c|c|c|c|}
\hline \multicolumn{3}{|c|}{ Nestlé } & \multicolumn{3}{|c|}{ La Universal } \\
\hline Variedad & $\begin{array}{l}\text { Peso } \\
\text { (gr) }\end{array}$ & $\begin{array}{l}\text { Precio de venta }(\$) \text { y } \\
\text { Precio unitario }(\$ / g r)\end{array}$ & Variedad & $\begin{array}{l}\text { Peso } \\
\text { (gr) }\end{array}$ & $\begin{array}{l}\text { Precio de venta }(\$) \text { y } \\
\text { Precio unitario (\$/gr) }\end{array}$ \\
\hline Negro & 100 & $1,59 \$ /(0,016 \$)$ & Negro y cacahuete L* & 100 & $1,47 \$ * /(0,015 \$)$ \\
\hline Chocolate Crunch & 80 & $1,69 \$ /(0,021 \$)$ & Negro y cacahuete $4 \mathrm{U}$ & 112 & $1,67 \$ /(0,015 \$)$ \\
\hline Blanco y arroz $4 \mathrm{U}$ & 80 & $1,70 \$ /(0,021 \$)$ & No presenta más variec & des en & la cartera de producto. \\
\hline \multicolumn{3}{|c|}{ Pacari } & \multicolumn{3}{|c|}{ Hershey's } \\
\hline Variedad & $\begin{array}{l}\text { Peso } \\
\text { (gr) }\end{array}$ & $\begin{array}{l}\text { Precio de venta }(\$) \text { y } \\
\text { Precio unitario (\$/gr) }\end{array}$ & Variedad & $\begin{array}{l}\text { Peso } \\
\text { (gr) }\end{array}$ & $\begin{array}{l}\text { Precio de venta (\$) y } \\
\text { Precio unitario (\$/gr) }\end{array}$ \\
\hline Negro (Esmeralda) & 50 & $1,94 \$ /(0,039 \$)$ & Negro 8U & 102 & $3,49 \$ /(0,034 \$)$ \\
\hline Negro (Maracuyá) & 50 & $1,94 \$ /(0,039 \$)$ & \multirow{2}{*}{\multicolumn{3}{|c|}{ No presenta más variedades en la cartera de producto. }} \\
\hline Negro (Arándano) & 50 & $1,94 \$ /(0,039 \$)$ & & & \\
\hline
\end{tabular}

Nota: *Precio de venta más bajo de las diferentes categorías de productos de chocolate. Fuente: elaboración propia a partir de la visita a establecimientos (2021). 
Tabla 6. Resumen de precios de las principales marcas de chocolate por lugar de compra (Comisariato Aki)

\begin{tabular}{|c|c|c|c|c|c|}
\hline \multicolumn{3}{|c|}{ Nestlé } & \multicolumn{3}{|c|}{ La Universal } \\
\hline Variedad & $\begin{array}{l}\text { Peso } \\
\text { (gr) }\end{array}$ & $\begin{array}{l}\text { Precio de venta }(\$) \text { y } \\
\text { Precio unitario }(\$ / g r)\end{array}$ & Variedad & $\begin{array}{l}\text { Peso } \\
\text { (gr) }\end{array}$ & $\begin{array}{l}\text { Precio de venta }(\$) \text { y } \\
\text { Precio unitario }(\$ / \text { gr) }\end{array}$ \\
\hline Negro & 100 & $1,66 \$ /(0,017 \$ / g r)$ & Negro y cacahuete & 100 & $1,88 \$ /(0,019 \$ / g r)$ \\
\hline Blanco y arroz & 90 & $1,77 \$ /(0,020 \$ / g r)$ & Negro y blanco* & 112 & $1,56 \$ * /(0,014 \$ / g r)$ \\
\hline Negro y arroz Crunch & 90 & $1,77 \$ /(0,020 \$ / g r)$ & Negro y cacahuete $4 U^{*}$ & 112 & $1,56 \$ * /(0,014 \$ / g r)$ \\
\hline Blanco y arroz 4U & 80 & $1,73 \$ /(0,022 \$ / \mathrm{gr})$ & No presenta más varied & es en 1 & artera de producto. \\
\hline \multicolumn{3}{|c|}{ Pacari } & \multicolumn{3}{|c|}{ Hershey's } \\
\hline Variedad & $\begin{array}{l}\text { Peso } \\
\text { (gr) }\end{array}$ & $\begin{array}{l}\text { Precio de venta }(\$) \text { y } \\
\text { Precio unitario }(\$ / \text { gr) }\end{array}$ & Variedad & $\begin{array}{l}\text { Peso } \\
\text { (gr) }\end{array}$ & $\begin{array}{l}\text { Precio de venta }(\$) \text { y } \\
\text { Precio unitario }(\$ / \text { gr) }\end{array}$ \\
\hline Negro (Esmeralda) & 50 & $2,15 \$ /(0,043 \$ / g r)$ & Negro & 102 & $\begin{array}{c}3,80 \$ \text { (Oferta: } 2,65 \$) / \\
(0,026 \$ / \text { gr })\end{array}$ \\
\hline Negro (Maracuyá) & 50 & $2,15 \$ /(0,043 \$ / g r)$ & Blanco & 113 & $\begin{array}{c}3,80 \text { (Oferta: } 3,23 \$) / \\
(0,029 \$ / \text { gr })\end{array}$ \\
\hline $\begin{array}{l}\text { Negro (Arándano) } \\
\text { No presenta más varie }\end{array}$ & 50 & $\begin{array}{l}2,15 \$ /(0,043 \$ / \text { gr }) \\
\text { cartera de producto. }\end{array}$ & \multicolumn{3}{|c|}{ No presenta más variedades en la cartera de producto. } \\
\hline
\end{tabular}

Nota: *Precio de venta más bajo de las diferentes categorías de productos de chocolate. Fuente: elaboración propia a partir de la visita a establecimientos (2021).

En cuanto a la distribución, la oferta que posee la marca global es máxima, de tal forma que la cobertura a la que aspira Nestlé es total, por lo que está presente en todos los formatos y puntos de venta y, además, tiene una buena visualización, lo que favorece la preferencia por la marca. Esta misma estrategia, de gran intensidad en el canal de distribución, es seguida por la marca La Universal. Tanto una como otra disponen de un amplio espacio en el lineal. La comunicación es otra de las variables en que Nestlé, gracias a la dimensión global de la marca, extiende su imagen en todas las áreas geográficas en las que está presente.

En suma, el examen de las variables de marketing ha permitido concluir que la marca Nestlé combina un posicionamiento global de la marca y una adaptación de sus programas de marketing (producto y precio) al mercado local de Ecuador. La investigación del consumidor nos puede ayudar a comprender mejor este resultado y a confirmar si la globalidad percibida por la marca Nestlé predispone a comprar este producto.

\subsubsection{Investigación del comportamiento del consumidor}

\section{- Hábitos de compra}

El estudio empírico realizado identifica dos resultados. En primer lugar, se trata de un producto con un nivel de consumo relativamente alto, de tal forma que un porcentaje importante de consumidores consumen una vez al mes o cada dos meses alguna de las categorías analizadas (tableta de chocolate blanco, negro, almendras, bombón, caramelos o confitería). En segundo lugar, conviene destacar la tasa de consumo de tabletas de chocolate blanco (15,82\%, una vez al mes). Así, casi un $16 \%$ de los consumidores de Ecuador compran tabletas de chocolate blanco una vez al mes (Tabla 7). También destaca el consumo de chocolate negro (11,49\%, una vez al mes) mientras que los bombones $(8,52 \%)$ y la tableta de almendras $(2,97 \%$, una vez cada tres meses) son de consumo más ocasional. Los valores están redondeados a dos decimales.

Estos datos son coherentes con el estudio descriptivo realizado, donde se destaca que la tableta de chocolate blanco presenta una tasa de consumo relativamente alta. Este dato revela que la marca 
Nestlé ha concentrado un gran esfuerzo comercial en desarrollar un producto adaptado a las preferencias de este mercado. De esta forma, ha desarrollado la marca de chocolate blanco Nestlé Galak, que contiene, además, arroz crocante. Asimismo, se señalizan en el producto los atributos directamente conectados con el motivo hedónico, como es el arroz crocante y el sabor dulce. La importancia del atributo hedónico en el consumo del chocolate ya ha sido explicada en la revisión de la literatura de los estudios del chocolate (Kuikka y Laukkanen, 2012; Ozretic-Dosen, Skare, Krupka, 2007; Poelmans y Rousseau, 2016; Rousseau, 2015). De igual forma, se observa que el chocolate negro tiene una demanda importante, mientras que el chocolate con almendras tiene una demanda más ocasional. En el caso de los bombones, la compra es de una vez al mes $(8,52 \%)$. Finalmente, la compra de caramelos y confitería se podría considerar de menor relevancia.

Tabla 7. Frecuencias relativas de hábitos de compra condicionadas al tipo de chocolate consumido

\begin{tabular}{lcccccccc}
\hline & $\begin{array}{c}\text { Una vez } \\
\text { al mes }\end{array}$ & $\begin{array}{c}\text { Una vez cada } \\
\text { dos meses }\end{array}$ & $\begin{array}{c}\text { Una vez } \\
\text { cada tres } \\
\text { meses }\end{array}$ & $\begin{array}{c}\text { Una vez } \\
\text { cada cuatro } \\
\text { meses }\end{array}$ & $\begin{array}{c}\text { Una o dos } \\
\text { veces cada } \\
\text { seis meses }\end{array}$ & $\begin{array}{c}\text { Una o dos } \\
\text { veces al } \\
\text { año }\end{array}$ & $\begin{array}{c}\text { Menos o } \\
\text { nunca }\end{array}$ & $\begin{array}{c}\text { Total } \\
\text { (n) }\end{array}$ \\
\hline Tableta blanco & $15,82 \%$ & $6,20 \%$ & $2,97 \%$ & $1,81 \%$ & $0,58 \%$ & $0,52 \%$ & $0,00 \%$ & $27,90 \%$ \\
Tableta negro & $11,49 \%$ & $14,98 \%$ & $5,49 \%$ & $2,58 \%$ & $0,97 \%$ & $1,23 \%$ & $0,13 \%$ & $36,87 \%$ \\
Tableta almendras & $2,91 \%$ & $3,10 \%$ & $2,97 \%$ & $1,03 \%$ & $0,77 \%$ & $0,00 \%$ & $0,13 \%$ & $10,91 \%$ \\
Bombón & $8,52 \%$ & $5,04 \%$ & $2,45 \%$ & $1,55 \%$ & $0,84 \%$ & $0,45 \%$ & $0,00 \%$ & $18,85 \%$ \\
Caramelos & $2,13 \%$ & $0,90 \%$ & $1,03 \%$ & $0,13 \%$ & $0,45 \%$ & $0,26 \%$ & $0,00 \%$ & $4,90 \%$ \\
Confitería & $0,90 \%$ & $0,00 \%$ & $0,00 \%$ & $0,00 \%$ & $0,00 \%$ & $0,00 \%$ & $0,00 \%$ & $0,90 \%$ \\
\hline
\end{tabular}

Fuente: elaboración propia a partir del cuestionario (2021).

\section{- Importancia de la marca}

El estudio de esta variable es crucial para analizar la performance de la marca. Con anterioridad al análisis de la compra y del consumo del chocolate, la marca ejerce una influencia notable en la compra de este producto (Ahmed, Zbib, Sikander y Gilbert Noujaim, 2012). Por ello, se revisaron con mayor detalle las dimensiones de la notoriedad, tanto la notoriedad espontánea como la notoriedad sugerida. En efecto, los resultados revelan que la marca Nestlé posee una gran notoriedad espontánea, duplicando a la marca doméstica más relevante en el mercado de Ecuador como es La Universal (Figura 2 y Tabla 8). Este resultado no solo refuerza el análisis realizado, sino que pone de manifiesto una relación directa entre la cuota de mercado y la notoriedad espontánea.

De esta forma, las marcas que tengan una mayor notoriedad van a tener una posición predominante en la mente del consumidor. Por tanto, estos datos revelan que Nestlé ostenta un gran reconocimiento social, que contiene todos los elementos de una marca fuerte y de una marca conocida (Cerviño Fernández, 2014). La marca Nestlé, gracias a la superioridad ejercida a través del posicionamiento global, influye de una forma determinante en la compra. Este resultado es coherente con el modelo expuesto, lo cual incide en cómo el posicionamiento de la marca ejerce una gran influencia en la performance, en este caso de la cuota de mercado.

Además, para el mercado del chocolate, los consumidores son leales a la marca, porque la asocian con parámetros determinados de calidad y sabor, lo que, unido a una experiencia previa positiva, hace que los consumidores no cambien a otras marcas (Ozretic-Dosen, Skare y Krupka, 2007). Este resultado refuerza la función de garantía que ejerce el nombre de marca (del Río Lanza, Vázquez Casielles e Iglesias Argüelles, 2001). Estos factores acentúan que la reintención de compra sea alta, lo que influye enormemente en la consecución de una elevada cuota de mercado.

Por otro lado, la marca La Universal tiene un buen reconocimiento entre los consumidores de Ecuador. Se percibe una marca que, gracias a su reputación y buen hacer, consigue un reconocimiento que va más allá del público y se centra en el producto, en este caso el chocolate. 


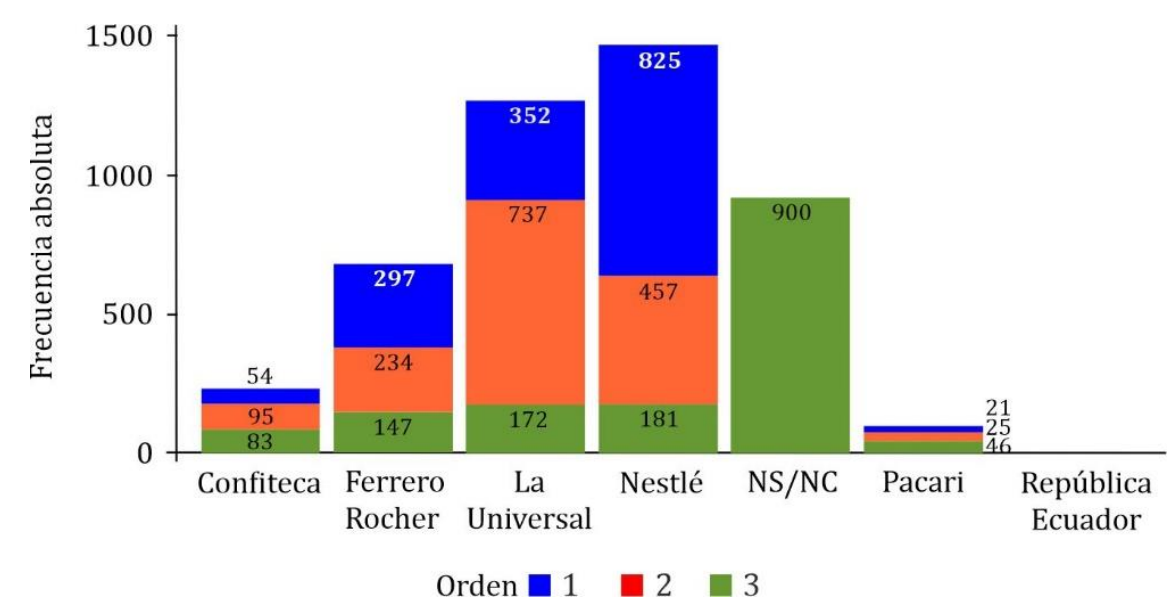

Figura 2. Frecuencias absolutas del orden de preferencia de las marcas más recordadas de productos de chocolate de acuerdo con el criterio de notoriedad espontánea. Fuente: elaboración propia a partir del cuestionario (2021).

Tabla 8. Frecuencias relativas del orden de preferencia de las marcas más recordadas de productos de chocolate de acuerdo con el criterio de notoriedad espontánea

\begin{tabular}{ccccccccc}
\hline & NS/NC & Nestlé & La Universal & Ferrero Rocher & Confiteca & Pacari & República del Cacao & Total \\
\hline $1^{\text {a }}$ & 0,00 & 53,26 & 22,72 & 19,17 & 3,49 & 1,36 & 0,00 & 100 \\
$2^{\text {a }}$ & 0,00 & 29,51 & 47,58 & 15,11 & 6,13 & 1,61 & 0,06 & 100 \\
$3^{\text {a }}$ & 59,39 & 11,69 & 11,10 & 9,49 & 5,36 & 2,97 & 0,00 & 100 \\
\hline
\end{tabular}

Fuente: elaboración propia a partir del cuestionario (2021).

\section{- Fuentes de valor de marca}

Como se puede comprobar, se trata de un mercado con un número reducido de marcas y en el que existe una marca dominante como es Nestlé, que lidera el mercado. Debido a que no se ha encontrado una fuente de datos que permita cuantificar con exactitud la cuota de mercado de las diferentes categorías de productos de chocolate para las diferentes marcas, se ha recurrido a una estimación de la cuota de mercado a partir del estudio realizado. Así, partiendo de los datos de la investigación de mercados, que permiten identificar la marca de productos de chocolate que habitualmente compran los consumidores de Ecuador, se obtiene la tasa de penetración. Esta misma aproximación fue utilizada por Romaniuk, Dawes y Nenycz-Thiel (2018). De esta manera, la marca Nestlé tendría una tasa de penetración de un 50,1\%, aproximadamente, frente a La Universal, su gran competidora, que alcanzaría un $24,98 \%$.

$\mathrm{Al}$ estudiar los valores de las diferentes dimensiones que componen el valor de marca, se observan tres resultados de interés. En primer lugar, las marcas de tabletas de chocolate que tienen una mayor cuota de mercado, como son Nestlé y su gran rival La Universal, son las que alcanzan la mayor notoriedad sugerida. El segundo resultado de interés que se puede comprobar en el análisis de la varianza (test de la $F$ ) es que no se observan diferencias significativas para el conjunto de las marcas en las puntuaciones de las dimensiones del valor de marca, como son la calidad percibida ( $p$-valor $=0,217)$, las asociaciones ( $p$-valor $=0,338)$ y la lealtad $(p$-valor $=0,115)$ (Tabla 9). Sin embargo, hay diferencias en las puntuaciones con respecto a la percepción de la notoriedad para al menos una marca $(p$-valor $<$ $0,05)$. Lo mismo ocurre con la disposición a pagar una prima en el precio. La marca La Universal presenta un valor significativamente más alto $(3,79)$ que Nestlé $(3,55)$, como podemos observar en la Tabla 10. Parece que la marca Nestlé se centra más en conseguir la mayor cuota de mercado posible en 
lugar de ofrecer un producto con una alta prima en precio. Por ello, se infiere que el resultado o performance tan alta de la marca Nestlé es consecuencia no solo de la acción comercial de la marca, sino del resultado conjunto de las variables de marketing-mix como la amplitud de gama, el precio o la promoción, la distribución o la comunicación y publicidad.

Tabla 9. Análisis de la varianza. Puntuaciones con respecto a las dimensiones del valor de marca

\begin{tabular}{llccccc}
\hline $\begin{array}{l}\text { Dimensión del } \\
\text { valor de marca y } \\
\text { prima en el precio }\end{array}$ & & $\begin{array}{c}\text { Grados de } \\
\text { libertad }\end{array}$ & Sum Sq & Mean Sq & F & Pr \\
\hline Calidad percibida & Factor & 4 & 1,7 & 0,4212 & 1,444 & $0,217^{*}$ \\
& Residuos & 1544 & 450,3 & 0,2916 & & \\
Notoriedad & Factor & 4 & 4,6 & 1,1566 & 4,782 & $0,00078^{* * *}$ \\
& Residuos & 1544 & 373,5 & 0,2419 & & \\
Asociaciones de & Factor & 4 & 0,88 & 0,2192 & 1,135 & $0,338^{*}$ \\
marca & Residuos & 1544 & 298,37 & 0,1932 & & \\
Lealtad & Factor & 4 & 2,9 & 0,7145 & 1,859 & $0,115^{*}$ \\
Ralor de marca & Fesiduos & 1544 & 593,5 & 0,3844 & & $0,0131^{* *}$ \\
Frima en el precio & Factor & 4 & 6,9 & 1,7215 & 3,175 & \\
& Residuos & 1544 & 837,1 & 0,5422 & & $3,99 \mathrm{e}-07^{* * *}$ \\
\hline
\end{tabular}

Nota: *no significativo $(p>0,05),{ }^{* *}$ diferencia significativa en las puntuaciones $(p<0,05),{ }^{* * *}$ diferencia significativa en las puntuaciones $(p<0,001)$. El término factor se refiere a cada uno de los grupos o marcas. Fuente: elaboración propia a partir del cuestionario (2021).

Tabla 10. Puntuación de las diferentes fuentes de valor de marca para las marcas habitualmente compradas de productos de chocolate.

\begin{tabular}{lccccccc}
\hline & Frecuencia & Notoriedad & $\begin{array}{c}\text { Calidad } \\
\text { percibida }\end{array}$ & Lealtad & $\begin{array}{c}\text { Asociaciones } \\
\text { de marca }\end{array}$ & $\begin{array}{c}\text { Valor de } \\
\text { marca }\end{array}$ & $\begin{array}{c}\text { Disposición a pagar } \\
\text { un precio primado }\end{array}$ \\
\hline Nestlé & 786 & 4,53 & 4,43 & 4,09 & 4,23 & 3,90 & 3,55 \\
Universal & 386 & 4,44 & 4,41 & 4,10 & 4,23 & 4,04 & 3,79 \\
Ferrero Rocher & 301 & 4,50 & 4,40 & 3,99 & 4,23 & 3,92 & 3,57 \\
Confiteca & 47 & 4,34 & 4,28 & 4,00 & 4,13 & 3,75 & 3,84 \\
Pacari & 29 & 4,27 & 4,28 & 4,07 & 4,11 & 3,98 & 3,40 \\
\hline
\end{tabular}

Fuente: elaboración propia a partir del cuestionario (2021).

\section{Discusión de resultados e implicaciones empresariales}

Esta investigación ha permitido dar respuesta al gap existente en la literatura de los estudios sobre la dualidad marca global y marca local en países en vías de desarrollo. El estudio, además, incorpora un doble punto de vista al incluir no solo el punto de vista de la oferta, en este caso de la empresa multinacional Nestlé, sino también el del consumidor. Desde un punto de vista teórico, el marco utilizado ha permitido dar respuesta a los objetivos y a las cuestiones planteadas. A la respuesta de si Nestlé estandariza o adapta, el estudio precisa que se trata de una estrategia híbrida que combina el posiciona- 
miento global, único para todo el mercado, con la adaptación de los programas de marketing de producto, precio o distribución al mercado doméstico de Ecuador.

El estudio realizado tiene importantes implicaciones para la gestión de las marcas globales. Este estudio ha puesto de manifiesto que, además de la marca, las diferentes variables de marketing-mix juegan un papel significativo en el desempeño de la empresa. Así, Nestlé se ha configurado como la empresa que lidera este mercado, no solo gracias al posicionamento global de la marca sino por una gestión adecuada de las variables del marketing-mix. Los principales pilares de esa estrategia se fundamentan en una amplia cartera de producto, con una amplia variedad y surtido de alternativas (chocolate negro, blanco, almendras); la fijación de precios competitivos, que intentan ajustarse a un nivel de renta bajo; una importante actividad promocional, con muy buena visualización en el lineal; y una estrategia de distribución intensiva con la que consigue una gran presencia en todos los formatos o tipos de establecimiento.

Otra de las implicaciones empresariales es el diseño de la estrategia de marketing internacional. Nestlé ha sabido adaptar una marca global a las características de un mercado local como Ecuador. Esta marca ha optado por concentrar grandes esfuerzos en comercializar chocolate blanco, sin olvidar la oferta de la clásica tableta de chocolate negro. A la tradicional oferta de la tableta de chocolate negro clásica, ha añadido otro producto que se adapta a las preferencias del mercado local como es el chocolate blanco con arroz crocante, el cual se acomoda muy bien a las preferencias de este mercado. La elección de los ingredientes de leche y arroz están inspirados en los hábitos culinarios y en la importancia de esa materia prima (arroz) en la dieta de los consumidores ecuatorianos. Tal y como señalan Özsomer y Altaras (2008), en la estrategia de marca global se combinan elementos de adaptación cultural y de superioridad global percibida de la marca, lo cual le permite conseguir un alto desempeño en el mercado. Esta superioridad global percibida por parte de los consumidores, conocida como efecto halo (Iglesias Argüelles, Vázquez Casielles y del Río Lanza, 2003), genera una actitud positiva e influye positivamente en la predisposición a comprar la marca por parte del consumidor final. Esta filosofía de marketing internacional y gestión local es lo que está permitiendo a la marca global conseguir una posición de liderazgo en el mercado del chocolate en un país de gran tradición de producción cacaotera como es Ecuador.

Otra importante implicación es el desempeño de la marca, que se concentra en la cuota de mercado en lugar del valor de marca. Nestlé no está buscando una prima en precio sino una mayor cuota de mercado. En efecto, tal y como ocurre con la variable valor de marca, la disposición a pagar una prima en el precio es significativamente inferior en el caso de la marca Nestlé, mientras que las marcas locales como Pacari o La Universal presentan valores más altos. Un patrón similar se observa para la marca de bombones Ferrero Rocher. Además, tanto la notoriedad espontánea como la notoriedad sugerida alcanzan valores altos.

Las marcas locales de Ecuador pueden aprovechar las ventajas de localización y el conocimiento del mercado por parte de los productores o cacaoteros. De esta forma, se puede incrementar la exportación de chocolate al exterior, donde factores como una denominación de origen o una certificación mundial tienen cada vez más relevancia (Pino Peralta, Aguilar Azuero y Sisalema Morejón, 2018). La denominación de origen Cacao Arriba exige aplicar prácticas orgánicas en la producción, lo que permitiría, además de proteger la producción, acceder a los mercados internacionales (Criterios digital, 2019).

\section{Conclusiones}

Una de las grandes cuestiones al abordar las marcas globales ha sido la de investigar si existe una estandarización completa de las actividades de marketing, o si la marca global se adapta a los mercados locales. Desde el punto de vista de la oferta, la marca piensa globalmente y actúa localmente, adaptando la oferta a las características particulares de una determinada área geográfica. Así, la marca 
Nestlé ha sabido adaptar el producto a las características del mercado de Ecuador. Además, inspirado en el nuevo enfoque de demanda, donde es posible identificar segmentos de consumidores globales, ha desarrollado un nuevo producto elaborado a partir de cacao fino.

Esta filosofía de gestión y de marketing internacional permite a la empresa consolidar su posición de liderazgo en un país que está entre los grandes productores de cacao a nivel mundial. Además, a través de la combinación óptima de las variables del marketing ha sabido adaptarse al mercado local. De este modo, por medio de una selección óptima de la amplitud de la cartera de producto, precios competitivos, promoción intensa (descuentos y promoción en cantidad) y distribución intensiva ha conseguido conectar con los consumidores de Ecuador, lo que le ha permitido liderar y consolidar la cuota de mercado. Por otro lado, existe una oportunidad para las marcas locales de exportar chocolate con un precio superior, puesto que el cacao de Ecuador está cada vez más valorado y apreciado en los mercados internacionales. Es necesario mejorar las capacidades comerciales de los agentes que operan a lo largo de la cadena de producción, capitalizar la imagen de calidad del cacao ecuatoriano e incrementar la innovación y desarrollo de nuevos métodos de producción y elaboración más sostenibles.

Como futuros trabajos exponemos diferentes líneas de interés como pueden ser la reorganización de la cadena de valor, la exportación a mercados con alto nivel de renta (Japón, Estados Unidos) y la innovación y desarrollo de nuevos métodos de producción del chocolate más sostenibles. Por último, se propone avanzar en la dualidad de marca global y local en entornos de incertidumbre, como es el que ha causado la reciente pandemia mundial (Ng, Faraji-Rad, Batra, 2021).

Por último, existen limitaciones que podrían afrontarse en las siguientes investigaciones. La cuota de mercado de las marcas ha sido estimada por una variable proxy como es la tasa de penetración de los mercados, por lo que es necesario incorporar, además, la rotación, lo que permitiría saber no solo el número de veces que acude al establecimiento sino también la cantidad adquirida en cada acto de compra. De igual manera, la investigación se podría enriquecer más si se incorpora el análisis de la actitud del consumidor hacia la globalidad de la marca, lo cual implicaría, además, analizar las actitudes etnocentristas.

\section{Autoría del trabajo}

Conceptualización, D.C.D., A.M. del C.P., y S.R.A.M.; metodología, D.C.D., A.M. del C.P. y S.R.A.M.; adquisición de datos, S.R.A.M.; análisis e interpretación: D.C.D., A.M. del C.P. y S.R.A.M.; redacción y preparación del borrador, D.C.D., A.M. del C.P. y S.R.A.M.; revisión y edición, D.C.D., A.M. del C.P. y S.R.A.M. Todos los autores han leído y están de acuerdo con la versión publicada del manuscrito.

\section{Agradecimientos}

Los autores agradecen el trabajo y los valiosos comentarios que han realizado dos revisores que han evaluado este estudo.

\section{Bibliografía}

Aaker, D. A. (1991). Managing brand equity: Capitalizing on the value of a brand name. New York, NY: The Free Press.

Aaker, D. A. (1996a). Building strong brands. New York, NY: The Free Press.

Aaker, D. A. (1996b). Measuring brand equity across products and markets. California Management Review, 38(3), 102-120.

Aaker, D. A., y Joachimsthaler, E. (1999). The lure of global branding. Harvard Business Review, 77(6), 137-144. Recuperado de: https://hbr.org/1999/11/the-lure-of-global-branding 
Ahmed, Z. U., Zbib, I. J., Sikander, A., y Gilbert Noujaim, R. (2012). Does country of brand origin (COBO) matter for the lebanese consumers? EuroMed Journal of Business, 7(2), 108-128. DOI: https://doi.org/10.1108/14502191211245561

Alden, D. L., Steenkamp, J.-B. E. M., y Batra, R. (1999). Brand positioning through advertising in Asia, North America, and Europe: The role of global consumer culture. Journal of Marketing, 63(1), 75-87. DOI: https://doi.org/10.1177/002224299906300106

ANECACAO. (2019). Estadísticas de exportación. Guayaquil, Ecuador: Asociación Nacional de Exportadores de Cacao de Ecuador. Recuperado de: http://www.anecacao.com/es/estadisticas/estadisticas-actuales.html

Calvo Dopico, D., del Castillo Puente, A. M., y Herrera Peña, J. N. (2020). Análisis del valor añadido de un nuevo envase en el mercado de azúcar y su influencia en la disposición a pagar. Revista Galega de Economía, 29(3), 1-17. DOI: https://doi.org/10.15304/rge.29.3.6705

Cerviño Fernández, J., y Baena Graciá, V. (2014). Nuevas dimensiones y problemáticas en el ámbito de la creación y gestión de marcas. Cuadernos de Estudios Empresariales, 24, 11-50.

DOI: https://doi.org/10.5209/rev CESE.2014.v24.48609

Cerviño Fernández, J., y Cebollada Calvo, J. J. (2005). Estandarización "versus" adaptación en los mercados internacionales: ¿todavía un dilema? Harvard Deusto Marketing y Ventas, 70, 36-41. Recuperado de: https://www.harvard-deusto.com/estandarizacion-versus-adaptacion-en-los-mercados-internacionalestodavia-un-dilema

Chabowski, B. R., Samiee, S., y Hult, T. M. (2013). A bibliometric analysis of the global branding literature and a research agenda. Journal of International Business Studies, 44(6), 622-634. DOI: https://doi.org/10.1057/JIBS.2013.20

Craig, C. S. y Douglas, S. P. (2000). Configural advantage in global markets. Journal of International Marketing, 8(1), 6-26. DOI: https://doi.org/10.1509/jimk.8.1.6.19564

Criterios digital. (2019). Cinco productos ecuatorianos cuentan con denominación única en el mundo. Quito, Ecuador: Cámara de Comercio de Quito. Recuperado de: https://criteriosdigital.com/datos/rcriterios/cincoproductos-ecuatorianos-cuentan-con-denominacion-unica-en-el-mundo/

del Río Lanza, A. B., Vázquez Casielles, R., e Iglesias Argüelles, V. (2001). El valor de marca: perspectivas de análisis y criterios de estimación. Cuadernos de Gestión, 1(2), 87-102.

Recuperado de: http://hdl.handle.net/10810/6997

Demont, M., Rutsaert, P., Ndour, M., Verbeke, W., Seck, P. A., \& Tollens, E. (2013). Experimental auctions, collective induction and choice shift: Willingness-to-pay for rice quality in Senegal. European Review of Agricultural Economics, 40(2), 261-286. DOI: https://doi.org/10.1093/erae/jbs021

Dimofte, C. V., Johansson, J. K., y Ronkainen, I. A. (2008). Spanning the globe. Marketing Management, 17(5), 40-43.

Fernández-Ferrín, P., Calvo-Turrientes, A., Bande, B., Artaraz-Miñón, M., y Galán-Ladero, M (2018). The valuation and purchase of food products that combine local, regional and traditional features: The influence of consumer ethnocentrism. Food Quality and Preference, 64(march), 138-147. DOI: https://doi.org/10.1016/j.foodqual.2017.09.015

Grunert, K. G., Baadsgaard, A., Larsen, H. H., y Madsen, T. K. (1996). Market orientation in food and agriculture: Summary, implications, and research perspectives. En K. G. Grunert, H. H. Larsen, T. K. Madsen y A. Baadsgaard, Market orientation in food agriculture (pp. 243-253). Boston, MA: Springer. DOI: https://doi.org/10.1007/978-1-4613-1301-4 12

Harsono, S., Perdana, S., Riyadi, D. B., y Normasita (2018). The influence of brand image, brand trust, perceived quality and perceived value on consumer purchase intention at different categories of product. Advanced Science Letters, 24 (5), 3198-3207. DOI: https://doi.org/10.1166/asl.2018.11343

Hassan, S. S., y Katsanis, L. P. (1991). Identification of global consumer segments: A behavioral framework. Journal of International Consumer Marketing, 3(2), 11-28. DOI: https://doi.org/10.1300/J046v03n02_03

Iglesias Argüelles, V., Vázquez Casielles, R., y del Río Lanza, A. B. (2003). La influencia de la marca en la evaluación del producto: consideraciones sobre el efecto halo. Revista Europea de Dirección y Economía de la Empresa, 12(3), 25-40.

INEC. (2020). Estadísticas agropecuarias. Quito, Ecuador: Instituto Nacional de Estadística y Censos. Recuperado de: https://www.ecuadorencifras.gob.ec/estadisticas-agropecuarias-2/

Kapferer, J.-N. (2005). The post-global brand. Brand Management, 12(5), 319-324. DOI: https://doi.org/10.1057/palgrave.bm.2540228

Keller, K. .L. (1993). Conceptualizing, measuring, and managing customer-based brand equity. Journal of Marketing, 57, 1-22. DOI: http://dx.doi.org/10.2307/1252054 
Keller, K. L. (2003). Strategic brand management. New Jersey, NJ: Prentice Hall.

Kilduff, K., y Núñez Tabales, J. M. (2017). Country branding and its effect on the consumer in the global market. Management Letters, 17(1), 83-104. DOI: https://doi.org/10.5295/cdg.150543kk

Klein, J. G. (2002). Us versus them, or us versus everyone? Delineating consumer aversion to foreign goods. Journal of International Business Studies, 33, 345-363. DOI: https://doi.org/10.1057/PALGRAVE.JIBS.8491020

Kuikka, A., y Laukkanen, T. (2012). Brand loyalty and the role of hedonic value. Journal of Product \& Brand Management, 21(7), 529-537. D0I: https://doi.org/10.1108/10610421211276277

Le, N.-H., Thi Nguyen, H.-M. T., y van Nguyen, T. (2013). National identity and the perceived values of foreign products with local brands: The case of local wine in Vietnam. Asia Pacific Journal of Marketing and Logistics, 25(5), 765-783. DOI: https://doi.org/10.1108/APJML-01-2013-0017

Leclerc, F., Schmitt, B. H., y Dubé, L. (1994). Foreign branding and its effects on product perceptions and attitudes. Journal of Marketing Research, 31(2), 263-270. DOI: https://doi.org/10.1177/002224379403100209

Levitt, T. (1983). The globalization of markets. Harvard Business Review, 61(3), 92-102. DOI: https://doi.org/10.1002/tie.5060250311

Lybeck, A., Holmlund-Rytkönen, M., y Sääksjärvi, M. (2006). Store brands vs. manufacturer brands: Consumer perception and buying of chocolate bars in Finland. The International Review of Retail Distribution and Consumer Research, 16(4), 471-492. DOI: https://doi.org/10.1080/09593960600844343

Ng, S., Faraji-Rad, A., y Batra, R. (2021). Uncertainty evokes consumers' preference for brands incongruent with their global-local citizenship identity. Journal of Marketing Research, 58(2), 400-415.

DOI: https://doi.org/10.1177/0022243720972956

Ozretic-Dosen, D., Skare, V., y Krupka, Z (2007). Assessments of country of origin and brand cues in evaluating a Croatian, Western and Eastern European food product. Journal of Business Research, 60(2), 130-136. DOI: https://doi.org/10.1016/j.jbusres.2006.10.011

Özsomer, A. (2012). The interplay between global and local brands: A closer look at perceived brand globalness and local iconness. Journal of International Marketing, 20(2), 72-95. DOI: https://doi.org/10.1509/jim.11.0105

Özsomer, A., y Altaras, S. (2008). Global brand purchase likelihood: A critical synthesis and an integrated conceptual framework. Journal of International Marketing, 16(4), 1-28. DOI: https://doi.org/10.1509/jimk.16.4.1

Pino Peralta, S. L., Aguilar Azuero, H. R., y Sisalema Morejón, L. A. (2018). La denominación de origen para cacao arriba. En busca del Santo Grial. Espacios, 39(16), 13-28.

Recuperado de: https://www.revistaespacios.com/a18v39n16/a18v39n16p13.pdf

Poelmans, E., y Rousseau, S. (2016). How do chocolate lovers balance taste and ethical considerations? British Food Journal, 118(2), 343-361. DOI: https://doi.org/10.1108/BFJ-06-2015-0208

Rajh, E., Vranesevic, T., y Tolic, D. (2003). Croatian food industry - brand equity in selected product categories. British Food Journal, 105(4/5), 263-273. DOI: https://doi.org/10.1108/00070700310477059

Romaniuk, J., Dawes, J., y Nenycz-Thiel, M. (2018). Modeling brand market share change in emerging markets. International Marketing Review, 35(5), 785-805. DOI: https://doi.org/10.1108/IMR-01-2017-0006

Rousseau, S. (2015). The role of organic and fair trade labels when choosing chocolate. Food Quality and Preference, 44(september), 92-100. DOI: https://doi.org/10.1016/j.foodqual.2015.04.002

Schuiling, I., y Kapferer, J.-N. (2004). Executive insights: Real differences between local and international brands: Strategic implications for international marketers. Journal of International Marketing, 12(4), 97-112. DOI: https://doi.org/10.1509/jimk.12.4.97.53217

Shimp, T. A., y Sharma, S. (1987). Consumer ethnocentrism: Construction and validation of the CETSCALE. Journal of Marketing Research, 24(3), 280-289. DOI: https://doi.org/10.2307/3151638

Steenkamp, J.-B. E. M. (1986). Perceived quality of food products and its relationship to consumer preferences: Theory and measurement. Journal of Food Quality, 9(6), 373-386.

DOI: https://doi.org/10.1111/j.1745-4557.1986.tb00807.x

Steenkamp, J.-B. E. M., Batra, R., y Alden, D. L. (2003). How perceived brand globalness creates brand value. Journal of International Business Studies, 34(1), 53-65. DOI: https://doi.org/10.1057/palgrave.jibs.8400002

Taylor, E. L., Quelch, J. A., y Holt, D. B. (2004). Cómo compiten las marcas globales. Harvard Deusto Business Review, 82(9), 68-75.

Thaichon, P., Jebarajakirthy, C., Tatuu, P., y Gautam Gajbhiyeb, R. (2018). Are you a chocolate lover? An investigation of the repurchase behavior of chocolate consumers. Journal of Food Products Marketing, 24(2), $163-176$. DOI: https://doi.org/10.1080/10454446.2017.1266551 
Winit, W., Gregory, G., Cleveland, M., y Verlegh, P. (2014). Global vs local brands: How home country bias and price differences impact brand evaluations. International Marketing Review, 31(2), 102-128.

DOI: https://doi.org/10.1108/IMR-01-2012-0001

Yoo, B., y Donthu, N. (2001). Developing and validating a multidimensional consumer-based brand. Journal of Business Research, 52(1), 1-14. DOI: https://doi.org/10.1016/S0148-2963(99)00098-3

Yoo, B., Donthu, N., y Lee, S. (2000). An examination of selected marketing mix elements and brand equity. Journal of the Academy of Marketing Science, 28, 195-211.

DOI: http://doi.org/10.1177/0092070300282002 